\title{
La hipertensión arterial no controlada y sus factores asociados en un programa de hipertensión
}

\author{
Uncontrolled hypertension and its associated factors in a hypertension program
}

\author{
Yenys M. Regino-Ruenes ${ }^{1 *}$, Mario A. Quintero-Velásquez² y Juan F. Saldarriaga-Franco ${ }^{3}$ \\ ${ }^{1}$ Facultad Nacional de Salud Pública, Universidad de Antioquia; ${ }^{2}$ Grupo de Investigación en Medicina Deportiva, Laboratorio de Fisiología Médica \\ - INDEPORTES, Antioquia; ${ }^{3}$ Grupo de Epidemiología, Facultad Nacional de Salud Pública, Universidad de Antioquia. Medellín, Colombia
}

\section{Resumen}

Objetivo: Identificar la proporción de hipertensos no controlados y sus factores asociados, en pacientes del programa de hipertensión arterial de Pueblorrico, Antioquia, 2018. Método: Estudio transversal de asociación, en el que se obtuvieron datos sociodemográficos, clínicos y antropométricos. Se aplicaron encuestas y la revisión de historias clínicas en una muestra de 272 pacientes. Se realizó la caracterización de los pacientes. Por regresión logística multivariada se obtuvieron las razones de disparidad y los intervalos de confianza del 95\% (IC95\%). Resultados: El no control de la presión arterial se evidenció en el 35.3\% (IC 95\%: 29.41-41.20). Las variables obesidad (odds ratio [OR]: 2.1; IC95\%: 1.05-4.31), utilizar más de tres medicamentos al día (OR: 2.0; IC95\%: 1.19-3.44) y asistir a menos de cuatro controles de seguimiento al año (OR: 2.2; IC95\%: 1.03-4.74) se asociaron de manera significativa como factores de riesgo para el no control de la presión arterial. Tener excelente percepción de la calidad del programa fue un factor protector (OR: 0.5; IC95\%: 0.28-0.87). Conclusiones: La proporción de pacientes hipertensos que no logran la meta terapéutica (presión arterial $\leq 140 / 90 \mathrm{~mm} \mathrm{Hg}$ ) continúa siendo un desafío para la práctica clínica y la salud pública. La adherencia a los controles y el control de factores de riesgo, como la obesidad, son intervenciones importantes en este contexto.

Palabras clave: Hipertensión arterial. Presión arterial. Índice terapéutico.

\section{Abstract}

Objective: To identify the proportion of uncontrolled hypertensive patients and their associated factors in a population attending an HBP program. Method: Cross-sectional association study. Sociodemographic, clinical and anthropometric data were obtained. Surveys and review of medical records were applied in a sample of 272 participants. The characterization of the patients was performed. By multivariate logistic regression the odds ratios (OR) and confidence intervals (95\% Cl) were obtained. Results: The non-control of blood pressure was evidenced in 35.3\% (95\% Cl: 29.41-41.20). The variables obesity (OR: 2.1; 95\% Cl: 1.05-4.31), use more than three medications per day (OR: 2.0; 95\% Cl: 1.19-3.44), and attending less than four follow-up controls per year (OR: 2.2; 95\% Cl: 1.03-4.74), were significantly associated as a risk factor for non-BP control. Having an excellent perception of the quality program was a protective factor (OR: $0.5 ; 95 \% \mathrm{Cl}$ : $0.28-0.87$ ). Conclusions: The proportion of hypertensive patients who do not achieve the therapeutic goal (PA $\leq 140 / 90 \mathrm{~mm} \mathrm{Hg}$ ), remains a challenge for clinical practice and public health. The adherence and control of risk factors such as obesity are important interventions in this context.

Key words: Hypertension. Blood pressure. Therapeutic index.

\section{Correspondencia:}

*Yenys M. Regino-Ruenes

E-mail: yenysregino@udea.edu.co
Disponible en internet: 07-01-2022 Rev Colomb Cardiol. 2021;28(6):648-655 www.rccardiologia.com 0120-5633 / @ 2020 Sociedad Colombiana de Cardiología y Cirugía Cardiovascular. Publicado por Permanyer. Este es un artículo open access bajo la licencia CC BY-NC-ND (http://creativecommons.org/licenses/by-nc-nd/4.0/). 


\section{Introducción}

La hipertensión arterial es una de las principales causas prevenibles de enfermedad cardiovascular y de muerte en el mundo. Su prevalencia global en adultos oscila entre el $30 \%$ y el $45 \%$, siendo mayor en hombres $(24 \%)$ que en mujeres (20\%). La alta prevalencia concuerda en todos los países, independientemente del estado de ingresos económicos de estos ${ }^{1}$. En Colombia, según la Encuesta Nacional de Salud 2007, de cada 100 personas entre 18 y 69 años, ocho refieren haber sido diagnosticadas como hipertensas en dos 0 más consultas; esta prevalencia disminuye al $6.7 \%$ cuando se tiene en cuenta el consumo de medicamentos antihipertensivos. No obstante, esta misma encuesta determinó una prevalencia del $22.8 \%$ de hipertensión arterial (cifras de presión arterial $\geq 140 / 90 \mathrm{mmHg}$ ) después de realizar una medición a una muestra representativa de la población encuestada ${ }^{2}$.

La hipertensión arterial es una enfermedad crónica, silenciosa, pues rara vez se acompaña de síntomas, lo que dificulta su diagnóstico y tratamiento. Se calcula que más de la mitad de los pacientes hipertensos desconocen que la padecen, lo cual retrasa el tratamiento precoz y eleva el riesgo de complicaciones y secuelas ${ }^{3}$.

La asociación epidemiológica entre la presión arterial y el riesgo de enfermedad cardiovascular se detecta desde niveles bajos de presión arterial. El aumento de $20 \mathrm{mmHg}$ en la presión sistólica y de $10 \mathrm{mmHg}$ en la diastólica (> 115/75 $\mathrm{mmHg}$ ) duplica el riesgo de muerte independientemente de otros factores, y para ambos sexos. Así, una reducción discreta de las cifras de presión arterial redunda en una disminución significativa del riesgo de enfermedad cardiovascular ${ }^{4,5}$.

Las tendencias mundiales del incremento de la expectativa de vida (en la mayoría de los países) ha propiciado que gran cantidad de personas transite al envejecimiento y, por ende, más personas padezcan hipertensión arterial. Esta enfermedad presenta una prevalencia del $60 \%$ en los mayores de 60 años ${ }^{1,6}$. Así, la transición demográfica es un factor necesario para comprender su comportamiento epidemiológico.

La hipertensión arterial rara vez ocurre de manera aislada y a menudo se relaciona con otros factores de riesgo, como tabaquismo, inactividad física, alimentación inadecuada, dislipidemia, intolerancia a la glucosa y obesidad; estos constituyen los principales factores de riesgo en la génesis de las enfermedades cardiovasculares, y de ahí su complejidad y la necesidad de manejo integral y control ${ }^{7,8}$.
El control de la presión arterial es un pilar fundamental para disminuir el riesgo de enfermedad cardiovascular y la mortalidad prematura; sin embargo, el logro de la meta terapéutica sigue siendo un problema en la población hipertensa en el mundo. Se estima que menos de la mitad de los pacientes diagnosticados y que reciben tratamiento logran un adecuado control de las cifras de presión arterial ${ }^{9,10}$. En diversas investigaciones se refieren numerosos factores como causas del inadecuado control de la presión arterial: el nivel socioeconómico, la baja adherencia al tratamiento farmacológico, la obesidad y la inercia médica; sin embargo, estos factores no son consistentes en poblaciones diferentes ${ }^{11,12}$. Por todo ello, resulta pertinente identificar la proporción de hipertensos no controlados y sus factores asociados, en las poblaciones que asisten a los programas de riesgo cardiovascular.

\section{Método}

Estudio transversal de asociación. Se seleccionó una muestra aleatoria de 272 pacientes, a partir de un universo de 924 pacientes inscritos en el programa de hipertensión arterial del Hospital San Vicente de Paúl, Municipio de Pueblorrico, Antioquia, Colombia, en 2018. Se incluyeron pacientes con diagnóstico de hipertensión arterial primaria con más de 12 meses de seguimiento en el programa, de ambos sexos y mayores de 18 años. Para el cálculo de la muestra se utilizó el programa Epi-Info Versión 7, con un nivel de confianza del 95\%, una proporción de hipertensión no controlada esperada del $32.5 \%^{3}$, un error máximo permisible del $5 \%$ y una sobreestimación del 10\% (anticipando datos perdidos).

Los datos fueron captados a través de la evaluación física del paciente, a cargo una enfermera profesional entrenada y con equipos calibrados. Las demás variables se obtuvieron de la aplicación de una encuesta y de la revisión de las historias clínicas, previa autorización del paciente mediante la firma del consentimiento informado.

\section{Variable principal (desenlace)}

Se consideró como no control de la presión arterial que el promedio de dos tomas de la presión arterial fuera $\geq 140 / 90 \mathrm{mmHg}$.

\section{Variables sociodemográficas}

Edad, sexo, estado civil, nivel educativo, lugar de residencia y nivel socioeconómico. 


\section{Medidas antropométricas y paraclínicas}

Peso, talla, perímetro de la cintura, índice de masa corporal y presión arterial (la toma se realizó con un esfigmomanómetro de mercurio calibrado, con el paciente sentado, en dos mediciones con un intervalo de 5 minutos).

Entre los exámenes de laboratorios se incluyeron colesterol total, lipoproteínas de alta densidad (HDL), lipoproteínas de baja densidad (LDL), triglicéridos y glucemia.

\section{Factores comportamentales $y$ comorbilidad}

Tabaquismo y consumo de alcohol (consumo actual), actividad física (inactividad: menos de 150 minutos de actividad física a la semana), adherencia al tratamiento farmacológico (según el test de Morisky-Green), tipo y cantidad de medicamentos por día, tipo de comorbilidad, variables identificadas a través de la encuesta y la revisión de la historia clínica.

\section{Factores del servicio de salud}

Número de controles en el año, profesional que realiza los controles, tiempo que tarde en llegar a la institución, percepción del paciente sobre el programa, educación en salud y recomendaciones brindadas.

El estudio contó con el aval del Comité de Ética de la Universidad de Antioquia, Facultad Nacional de Salud Pública (Cl 00238-2018), en la categoría de investigación de riesgo mayor al mínimo.

Se verificó el supuesto de normalidad con la prueba de Kolmogórov-Smirnov. Por medio de análisis univariado se describen las características de los pacientes. Las variables cuantitativas se presentan según la media y la desviación estándar o la mediana y el rango intercuartílico. Posteriormente se efectuó el análisis bivariado para identificar las asociaciones simples, y las variables candidatas para el modelo multivariado de asociación, considerando el criterio Hosmer-Lemeshow $(p<0.25)$, las evidencias de otros estudios, la plausibilidad y el criterio del experto. Se utilizó la prueba chi al cuadrado para la asociación entre variables cualitativas, y las pruebas $t$ de Student $O U$ de Mann-Whitney para la comparación de variables cuantitativas (según su distribución). Finalmente, se construyó un modelo de regresión logística binaria multivariada para calcular la razón de disparidad (OR, odds ratio) y los intervalos de confianza del 95\%
(IC95\%), con el fin de estimar los factores asociados al no control de la presión arterial. El nivel de significación establecido fue del $5 \%(\alpha: 0.05)$. El software empleado para los análisis estadísticos fue el SPSS Versión 22.

\section{Resultados}

En los 272 pacientes, la edad promedio fue de $56 \pm$ 14 años. El $67.3 \%$ eran de sexo femenino. Más de la mitad pertenecían al área urbana, con predominio de los estratos 1 y 2 , y el nivel educativo preponderante fue básica primaria. Respecto a los factores de riesgo, se encontró que la mayor prevalencia corresponde a la inactividad física, seguida del tabaquismo y el consumo de licor; también se evidencia un porcentaje significativo de pacientes que toman más de tres medicamentos por día y, a su vez, un alto porcentaje de pacientes que no son adherentes al tratamiento antihipertensivo prescrito en el programa. Más de la mitad de los pacientes presentan algún grado de sobrepeso u obesidad (Tabla 1).

Se halló que la tercera parte de los pacientes del programa no alcanzan la meta de control de la presión arterial (35.2\%). Al separar la población de no controlados por edad y sexo, se encontró que el mayor porcentaje de no control corresponde a las mujeres menores de 60 años (Fig. 1).

Sumadas a la hipertensión arterial, la más predominante fue la dislipidemia, seguida de la diabetes mellitus (Fig. 2).

Luego de ajustar por covariables en el análisis de regresión logística, se identificó que las variables asociadas significativamente con el no control de la presión arterial fueron la obesidad (OR: 2.1; IC95\%: 1.05-4.31), utilizar más de tres medicamentos al día (OR: 2.0; IC95\%. 1.19-3.44) y asistir a menos de cuatro controles de seguimiento al año (OR: 2.2; IC95\%: 1.034.74). Tener una excelente percepción de la calidad del programa de hipertensión arterial se halló como un factor protector (OR: 0.5; IC95\%: 0.28-0.87) (Tabla 2).

\section{Discusión}

Este estudio identificó una proporción de hipertensos no controlados del $35.3 \%$, situación similar a la encontrada en investigaciones realizadas en Canadá y Alabama, en las que los participantes tenían mal control de la hipertensión arterial en un $38.5 \%$ y un $33.9 \%$, respectivamente ${ }^{13,14}$. En contraste, otros estudios realizados en los Estados Unidos de América, Irán y 
Tabla 1. Características sociodemográficas y clínicas de los pacientes del programa de hipertensión del Hospital San Vicente de Paúl, Municipio de Pueblorrico, Antioquia, 2018.

\begin{tabular}{|c|c|c|c|c|c|}
\hline & \multicolumn{2}{|c|}{ Presión arterial controlad } & \multicolumn{2}{|c|}{ Presión arterial no controlada } & \multirow{2}{*}{$\begin{array}{c}\text { Total } \\
\mathrm{n}=\mathbf{2 7 2}\end{array}$} \\
\hline & $n=176$ & $\%$ & $\mathrm{n}=96$ & $\%$ & \\
\hline $\begin{array}{l}\text { Sexo } \\
\text { Hombre } \\
\text { Mujer }\end{array}$ & $\begin{array}{c}61 \\
115\end{array}$ & $\begin{array}{l}34.7 \\
65.3\end{array}$ & $\begin{array}{l}28 \\
68\end{array}$ & $\begin{array}{l}29.2 \\
70.8\end{array}$ & $\begin{array}{c}89 \\
183\end{array}$ \\
\hline Edad (años, DE)* & & & & & $56 \pm 14$ \\
\hline $\begin{array}{l}\text { Estrato } \\
1 \\
2 \\
3\end{array}$ & $\begin{array}{l}75 \\
91 \\
10\end{array}$ & $\begin{array}{r}42.6 \\
51.7 \\
5.7\end{array}$ & $\begin{array}{c}48 \\
43 \\
5\end{array}$ & $\begin{array}{c}50 \\
44.8 \\
5.2\end{array}$ & $\begin{array}{c}123 \\
134 \\
15\end{array}$ \\
\hline $\begin{array}{l}\text { Ubicación } \\
\text { Urbano } \\
\text { Rural }\end{array}$ & $\begin{array}{c}108 \\
68\end{array}$ & $\begin{array}{l}61.4 \\
38.6\end{array}$ & $\begin{array}{l}55 \\
41\end{array}$ & $\begin{array}{l}57.3 \\
42.7\end{array}$ & $\begin{array}{l}163 \\
109\end{array}$ \\
\hline $\begin{array}{l}\text { Nivel educativo } \\
\text { Sin escolaridad } \\
\text { Primaria incompleta } \\
\text { Primaria completa } \\
\text { Secundaria } \\
\text { Secundaria complet } \\
\text { Técnico } \\
\text { Universidad }\end{array}$ & $\begin{array}{c}18 \\
101 \\
23 \\
15 \\
15 \\
3 \\
1\end{array}$ & $\begin{array}{c}10.2 \\
57.4 \\
13.1 \\
8.5 \\
8.5 \\
1.7 \\
0.6\end{array}$ & $\begin{array}{c}6 \\
65 \\
13 \\
3 \\
8 \\
0 \\
1\end{array}$ & $\begin{array}{c}6.3 \\
67.7 \\
13.6 \\
3.1 \\
8.3 \\
0 \\
1\end{array}$ & $\begin{array}{c}24 \\
166 \\
36 \\
18 \\
23 \\
3 \\
2\end{array}$ \\
\hline $\begin{array}{l}\text { Estado civil } \\
\text { Soltero } \\
\text { Casado } \\
\text { Viudo } \\
\text { Divorciado } \\
\text { Unión libre } \\
\text { Separado }\end{array}$ & $\begin{array}{c}22 \\
77 \\
41 \\
0 \\
21 \\
15\end{array}$ & $\begin{array}{c}12.5 \\
43.7 \\
23.3 \\
0 \\
12 \\
8.5\end{array}$ & $\begin{array}{c}14 \\
49 \\
14 \\
1 \\
10 \\
8\end{array}$ & $\begin{array}{c}14.6 \\
51 \\
14.6 \\
1 \\
10.4 \\
8.4\end{array}$ & $\begin{array}{c}36 \\
126 \\
55 \\
1 \\
31 \\
23\end{array}$ \\
\hline $\begin{array}{l}\text { Fuma } \\
\text { No } \\
\text { Sí }\end{array}$ & $\begin{array}{c}159 \\
17\end{array}$ & $\begin{array}{c}90.3 \\
9.7\end{array}$ & $\begin{array}{l}86 \\
10\end{array}$ & $\begin{array}{l}89.6 \\
10.4\end{array}$ & $\begin{array}{c}245 \\
27\end{array}$ \\
\hline $\begin{array}{l}\text { Alcohol } \\
\text { No } \\
\text { Sí }\end{array}$ & $\begin{array}{c}164 \\
12\end{array}$ & $\begin{array}{c}93.2 \\
6.8\end{array}$ & $\begin{array}{c}89 \\
7\end{array}$ & $\begin{array}{c}92.7 \\
7.3\end{array}$ & $\begin{array}{c}253 \\
19\end{array}$ \\
\hline $\begin{array}{l}\text { Actividad física } \\
\text { Activo } \\
\text { Inactivo }\end{array}$ & $\begin{array}{c}50 \\
126\end{array}$ & $\begin{array}{l}28.4 \\
71.6\end{array}$ & $\begin{array}{l}25 \\
71\end{array}$ & $\begin{array}{l}26 \\
74\end{array}$ & $\begin{array}{c}75 \\
197\end{array}$ \\
\hline $\begin{array}{l}\text { Adherencia } \\
\text { Sí } \\
\text { No }\end{array}$ & $\begin{array}{c}38 \\
138\end{array}$ & $\begin{array}{l}21.6 \\
78.4\end{array}$ & $\begin{array}{l}15 \\
81\end{array}$ & $\begin{array}{l}15.6 \\
84.4\end{array}$ & $\begin{array}{c}53 \\
219\end{array}$ \\
\hline $\begin{array}{l}\text { Recibe educación/recomendaciones } \\
\mathrm{Si} \\
\text { No }\end{array}$ & $\begin{array}{c}163 \\
13\end{array}$ & $\begin{array}{c}92.6 \\
7.4\end{array}$ & $\begin{array}{c}93 \\
3\end{array}$ & $\begin{array}{c}96.8 \\
3.2\end{array}$ & $\begin{array}{c}256 \\
16\end{array}$ \\
\hline $\begin{array}{l}\text { Polimedicado (usar más de } 3 \\
\text { medicamentos) } \\
\text { Sí } \\
\text { No }\end{array}$ & $\begin{array}{c}70 \\
106\end{array}$ & $\begin{array}{l}39.8 \\
60.2\end{array}$ & $\begin{array}{l}52 \\
44\end{array}$ & $\begin{array}{l}54.2 \\
45.8\end{array}$ & $\begin{array}{l}122 \\
150\end{array}$ \\
\hline $\begin{array}{l}\text { IMC } \\
\text { Normal } \\
\text { Bajo peso } \\
\text { Sobrepeso } \\
\text { Obesidad }\end{array}$ & $\begin{array}{l}62 \\
5 \\
69 \\
40\end{array}$ & $\begin{array}{c}35.3 \\
2.8 \\
39.2 \\
22.7\end{array}$ & $\begin{array}{c}25 \\
4 \\
34 \\
33\end{array}$ & $\begin{array}{c}26 \\
4.2 \\
35.4 \\
34.4\end{array}$ & $\begin{array}{c}87 \\
9 \\
103 \\
73\end{array}$ \\
\hline
\end{tabular}


Tabla 1. Características sociodemográficas y clínicas de los pacientes del programa de hipertensión del Hospital San Vicente de Paúl, Municipio de Pueblorrico, Antioquia, 2018 (continuación).

\begin{tabular}{|c|c|c|c|c|c|}
\hline & \multicolumn{2}{|c|}{ Presión arterial controlad } & \multicolumn{2}{|c|}{ Presión arterial no controlada } & \multirow{2}{*}{$\frac{\text { Total }}{n=272}$} \\
\hline & $n=176$ & $\%$ & $n=96$ & $\%$ & \\
\hline $\begin{array}{l}\text { Percepción } \\
\text { Excelente } \\
\text { Buena } \\
\text { Regular }\end{array}$ & $\begin{array}{c}50 \\
120 \\
6\end{array}$ & $\begin{array}{c}28.4 \\
68.2 \\
3.4\end{array}$ & $\begin{array}{l}30 \\
50 \\
16\end{array}$ & $\begin{array}{l}31.2 \\
52.1 \\
16.7\end{array}$ & $\begin{array}{c}80 \\
170 \\
22\end{array}$ \\
\hline $\begin{array}{l}\text { Número de controles por año } \\
4 \text { o más controles } \\
\text { Menos de } 4 \text { controles }\end{array}$ & $\begin{array}{l}112 \\
64\end{array}$ & $\begin{array}{l}63.6 \\
35.3\end{array}$ & $\begin{array}{l}49 \\
47\end{array}$ & $\begin{array}{l}51.1 \\
48.9\end{array}$ & $\begin{array}{l}161 \\
111\end{array}$ \\
\hline $\begin{array}{l}\text { Tiempo que tarda en llegar a la institución } \\
\text { (minutos) })^{* *}\end{array}$ & \multicolumn{2}{|c|}{$10(8-30)$} & \multicolumn{2}{|c|}{$15(5-35)$} & $13(6-30)$ \\
\hline Colesterol total $(\mathrm{mg} / \mathrm{dl})^{* *}$ & \multicolumn{2}{|c|}{$213(185-245)$} & \multicolumn{2}{|c|}{$213(187-239)$} & $213(185-245)$ \\
\hline $\mathrm{HDL}(\mathrm{mg} / \mathrm{dl})^{* *}$ & \multicolumn{2}{|c|}{$50(40-57)$} & \multicolumn{2}{|c|}{$47(41-55)$} & $48(40-56)$ \\
\hline Triglicéridos $(\mathrm{mg} / \mathrm{dl})^{* *}$ & \multicolumn{2}{|c|}{$154(108-202)$} & \multicolumn{2}{|c|}{133 (101-181) } & $143(103-196)$ \\
\hline Glicemia $(\mathrm{mg} / \mathrm{dl})^{* *}$ & \multicolumn{2}{|c|}{$95(86-106)$} & \multicolumn{2}{|c|}{97 (89-109) } & $96(96-108)$ \\
\hline $\mathrm{LDL}(\mathrm{mg} / \mathrm{dl} \mathrm{DE})^{*}$ & \multicolumn{2}{|c|}{133 DE 43} & \multicolumn{2}{|c|}{134 DE 39} & $134 \pm 41$ \\
\hline
\end{tabular}

DE: desviación estándar; HDL: lipoproteína de alta densidad; IMC: índice de masa corporal; LDL: lipoproteína de baja densidad.

*Valores expresados en media y desviación estándar.

**Valores expresados en mediana y rango intercuartílico.

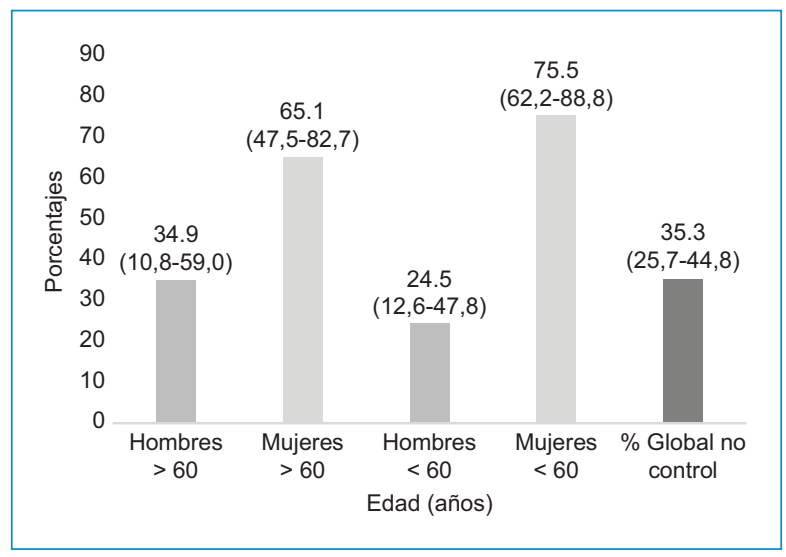

Figura 1. Proporción de pacientes hipertensos no controlados del programa, e intervalos de confianza, según sexo y edad. ESE Hospital San Vicente de Paúl, Pueblorrico, Antioquia, 2018.

Líbano reportaron que más de la mitad de los adultos con hipertensión no controlan sus valores de presión

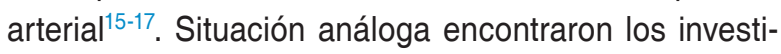
gadores del estudio PURE, en el que participaron 17 países de ingresos altos, medios y bajos, y reportaron que el $67.5 \%$ de los participantes correspondían a hipertensos no controlados ${ }^{3}$. Esta situación hace evidente que el mal control de la presión arterial es un

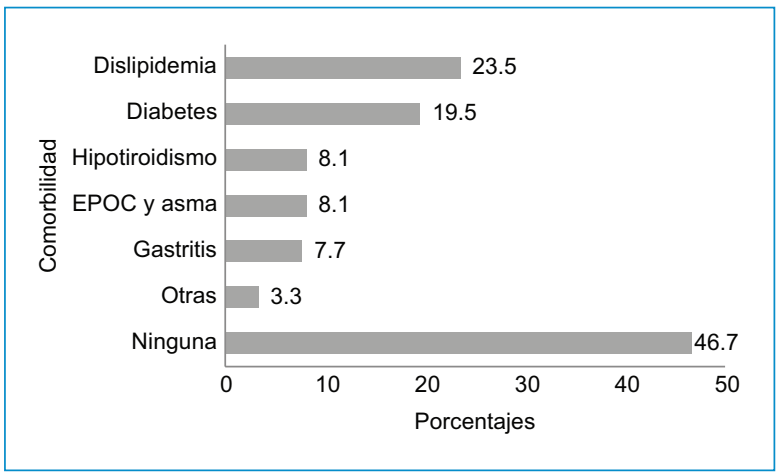

Figura 2. Comorbilidad en los pacientes hipertensos del programa de hipertensión del ESE Hospital San Vicente de Paúl, Pueblorrico, Antioquia, 2018.

problema de salud pública, con cifras críticas en todo el mundo, y que, por tanto, requiere el diseño de nuevas estrategias con trabajo articulado entre pacientes, cuidadores, sistema y personal de salud.

El cumplimiento del tratamiento farmacológico (adherencia) constituye un punto clave para el control de enfermedades crónicas como la hipertensión arterial ${ }^{18}$; sin embargo, en la población estudiada se evidenció una baja adherencia al tratamiento (19.5\%), resultado que concuerda con los de otras investigaciones ${ }^{19,20}$. Las razones para la falta de adherencia son 
Tabla 2. Modelo de regresión logística: factores asociados al no control de la presión arterial en pacientes del programa de hipertensión del Hospital San Vicente de Paúl, Pueblorrico, Antioquia, 2018

\begin{tabular}{|c|c|c|c|c|c|c|c|c|}
\hline & B & Error estándar & Wald & $\mathbf{g l}$ & Sig. & $\operatorname{Exp}(B)$ & Inferior & Superior \\
\hline Adherencia (no) & 0.489 & 0.352 & 1.931 & 1 & 0.165 & 1.630 & 0.818 & 3.249 \\
\hline Percepción (excelente) & -0.706 & 0.287 & 6.037 & 1 & 0.014 & 0.494 & 0.281 & 0.867 \\
\hline IMC (sobrepeso) & 0.234 & 0.330 & 0.504 & 1 & 0.478 & 1.264 & 0.662 & 2.412 \\
\hline IMC (obesidad) & 0.753 & 0.361 & 4.348 & 1 & 0.037 & 2.123 & 1.046 & 4.307 \\
\hline Polimedicados $(\geq 3)$ & 0.704 & 0.271 & 6.746 & 1 & 0.009 & 2.022 & 1.189 & 3.440 \\
\hline Controles $(<4)$ & 0.782 & 0.395 & 3.929 & 1 & 0.047 & 2.186 & 1.029 & 4.739 \\
\hline Sexo (hombre) & 0.034 & 0.307 & 0.012 & 1 & 0.912 & 1.035 & 0.567 & 1.888 \\
\hline Edad ( $\leq 60$ años) & 0.138 & 0.275 & 0.251 & 1 & 0.616 & 1.148 & 0.670 & 1.966 \\
\hline Constante & -1.398 & 0.500 & 7.800 & 1 & 0.005 & 0.247 & & \\
\hline
\end{tabular}

IC95\% para Exp (B).

IC95\%: intervalo de confianza del 95\%; IMC: índice de masa corporal.

Categorías de referencia: ser adherente, percepción regular del servicio, IMC normal, usar menos de tres medicamentos al día, asistir a cuatro o más controles, ser mayor de 60 años, sexo femenino.

multifactoriales $y$, con frecuencia, obedecen a una combinación de factores relacionados no solo con el paciente, sino además con el médico tratante, la familia, el servicio y el sistema de salud. Esta situación exige monitorización y compromiso en el marco del seguimiento de los programas de riesgo cardiovascular.

El $53.3 \%$ de los pacientes del estudio presentaron una o más comorbilidad sumada a la hipertensión arterial, hallazgo que también concuerda con los resultados de otros estudios ${ }^{7,21}$. Con mayor frecuencia, la hipertensión arterial tiende a estar acompañada de otros diagnósticos y de factores de riesgo (modificables y no modificables), tales como obesidad, inactividad física, tabaquismo y malos hábitos nutricionales, que en su conjunto aumentan el riesgo cardiovascular y dificultan el manejo adecuado, el tratamiento y el control de la hipertensión.

La asociación entre la obesidad y la hipertensión arterial no controlada se ha demostrado en estudios anteriores ${ }^{6,12,22}$. Esta puede estar explicada en la medida en que la obesidad induce mecanismos fisiopatológicos, como la retención de sodio y de agua, y el aumento del gasto cardiaco, que desempeñan un papel importante en la patogénesis de la hipertensión, lo cual aumenta el nivel de dificultad para el tratamiento y el control satisfactorio de las cifras de presión arterial. Por lo anterior, se puede considerar que trabajar sobre la pérdida de peso en los pacientes hipertensos-obesos debe ser una medida no farmacológica de primera línea para lograr la consecución de la meta terapéutica.

En este estudio, la polimedicación se asoció como factor de riesgo del no control de la presión arterial, hallazgo que concuerda con otros resultados ${ }^{23-25}$. La polimedicación en algunos pacientes hipertensos se presenta por dos grandes razones: 1) cuando el tratamiento con monoterapia es insuficiente, por lo que se hace necesario el uso de una combinación de medicamentos para el control de las cifras de presión arterial, tal como sugieren las sociedades científicas a través de sus guías clínicas; y 2) para el manejo de la comorbilidad identificada en los pacientes (en muchas ocasiones en estadios avanzados). Paralelamente debe considerarse que, a mayor número de medicamentos que tenga que tomar el paciente, mayor será el riesgo de interacción medicamentosa y efectos secundarios, y mayor tendencia a olvidar o confundir los tratamientos; por consiguiente, el riesgo de una menor adherencia al tratamiento antihipertensivo y, con ello, un menor control de la presión arterial ${ }^{26}$. Según lo anterior, la simplificación de la farmacoterapia, en cuanto a cantidad y frecuencia de dosificación, es un aspecto que puede favorecer la adherencia y mejorar el control de la presión arterial en los pacientes con hipertensión arterial.

En las instituciones de salud, los pacientes hipertensos acuden a citas programadas para su valoración y 
seguimiento, con el objetivo de lograr un control efectivo y sostenido de la presión arterial y sus factores de riesgo. Según los resultados de esta investigación, asistir a menos de cuatro citas de seguimiento por año es un factor de riesgo para el no control de la hipertensión arterial. Resultados similares evidenciaron Barreto, et al. ${ }^{23}$ en un estudio en Brasil en el año 2016. La asistencia frecuente a las citas de control permite un seguimiento detallado para la modificación de los factores de riesgos, la determinación de la condición actual del paciente y el análisis del comportamiento de la presión arterial. Además, facilita la educación en salud sobre los cambios requeridos en el estilo de vida y el autocuidado, la evaluación y el fortalecimiento en el cumplimiento de la terapia farmacológica, y por último, la identificación temprana de complicaciones. Esto tampoco quiere decir que, por sí sola, la asistencia a las citas programadas sea suficiente para el adecuado control de la hipertensión arterial.

Por otra parte, se halló que tener una excelente percepción de la calidad del programa (opinión del paciente) es un factor que reduce la posibilidad de no control de la presión arterial en un $50 \%$. Esta situación puede estar explicada por un mayor grado de cercanía con el paciente, la monitorización continua, las orientaciones permanentes y el apoyo de los profesionales, lo cual permite a los pacientes un mejor acercamiento a su enfermedad y los objetivos del programa, y con ello la adopción de prácticas para el cuidado de su salud ${ }^{27}$.

Entre las limitaciones del estudio se debe tener en cuenta que no se midieron factores que podrían ser relevantes en el control de la presión arterial, como el patrón nutricional de los pacientes, los hábitos de sueño, el estrés, el nivel de conocimiento de los profesionales de la salud sobre el manejo de la hipertensión y la inercia médica, etc. También debe considerarse que la población objeto de estudio eran adultos de un hospital de primer nivel, por lo cual las conclusiones no son extrapolables a otros niveles de atención. Igualmente, pudo haberse presentado un sesgo de selección, dado que quienes acuden al programa pueden proceder en mayor medida del casco urbano o del área rural cercana del municipio, subestimando así la magnitud del problema estudiado, por limitaciones de acceso geográfico.

En este estudio transversal de asociación se tienen limitaciones para la demostración de la causalidad, dado que mide exposiciones y el desenlace en un único momento del tiempo. En paralelo, deben considerarse las limitaciones relacionadas con la medición de los factores comportamentales, como tabaquismo, consumo de alcohol, adherencia farmacológica y actividad física, por medio de cuestionario con autorreporte del paciente, situación que puede generar sesgos de información que comprometen la validez de los datos e impiden evaluar su posible asociación con el no control de la hipertensión arterial. Otra limitante a considerar es que la calidad del programa se midió por la percepción que los pacientes tienen del programa de hipertensión arterial, y no por un estudio detallado de recursos, talento humano, procesos y resultados en salud del programa.

Los resultados señalan la importancia de diseñar indicadores y establecer metas que permitan evaluar aspectos clave, como la inasistencia a las citas programas, la adherencia al tratamiento farmacológico, el cumplimiento de las recomendaciones médicas, las modificaciones en el estilo de vida, la reducción del peso corporal (porcentaje de grasa), los efectos de la polimedicación y las posibles interacciones medicamentosas. Así, será fundamental emplear los resultados de los indicadores para evaluar la efectividad del programa y hacer los ajustes del caso, considerando un modelo de seguimiento clínico-epidemiológico. Otra estrategia radica en promover el empoderamiento de los pacientes en cuanto a la enfermedad, el cuidado y su control; este es un eslabón que exige trabajo para la oferta de un modelo renovado para el cuidado del paciente crónico. Finalmente, cobra relevancia el diseño de estrategias de intervención que involucren de manera activa al paciente, los cuidadores, la familia y los equipos interdisciplinarios en salud, con énfasis en la importancia de la modificación de los factores de riesgo (gestión individualizada) y el logro de las metas terapéuticas; esto deberá ser el foco de investigaciones a futuro.

\section{Conclusiones}

La proporción de pacientes hipertensos que no logran las metas de control de la presión arterial ( $\leq$ $140 / 90 \mathrm{~mm} \mathrm{Hg}$ ) fue del $35.3 \%$, lo cual evidencia que el control de la hipertensión arterial sigue siendo un desafío para la práctica clínica y la salud pública.

La adherencia a los programas orientados a la atención de los pacientes crónicos es una prioridad. La asistencia por sí sola no garantiza un control adecuado del paciente, y de ahí la importancia de la calidad de la oferta desde un equipo interdisciplinario, que posibilite acciones efectivas de control y prevención de complicaciones.

La identificación y el control de los factores de riesgo, como es el caso de la obesidad, debe ser un pilar 
fundamental en la atención clínica que se brinda a los pacientes.

Se requiere planificar estrategias novedosas que integren al paciente, los cuidadores, los servicios y los profesionales en salud, con miras a la intervención y la modificación de los factores de riesgo y el logro de las metas terapéuticas.

\section{Agradecimientos}

A la ESE Hospital San Vicente de Paúl, a la Facultad Nacional de Salud Pública de la Universidad de Antioquia. Los autores agradecen a los asesores y demás profesores que hicieron parte de sus procesos de formación académica y humana. Al Grupo de Epidemiología - Estrategia de Sostenibilidad CODI-2018; UdeA.

\section{Financiamiento}

No se tuvo ningún financiamiento.

\section{Conflicto de intereses}

Se declara no tener conflicto de intereses.

\section{Responsabilidades éticas}

Protección de personas y animales. Los autores declaran que para esta investigación no se han realizado experimentos en seres humanos ni en animales.

Confidencialidad de los datos. Los autores declaran que han seguido los protocolos de su centro de trabajo sobre la publicación de datos de pacientes.

Derecho a la privacidad y consentimiento informado. Los autores han obtenido el consentimiento informado de los pacientes y/o sujetos referidos en el artículo. Este documento obra en poder del autor de correspondencia.

\section{Bibliografía}

1. Williams B, Mancia G, Mancia G, Spiering W, Agabiti Rosei E, Azizi M, et al. $2018 \mathrm{ESC} / \mathrm{ESH}$ Guidelines for the management of arterial hypertension. Eur Heart J. 2018;39:3021-104.

2. Rodríguez J, Ruiz F, Peñaloza E, Eslava J, Gómez L, Sánchez H, et al. Encuesta Nacional de Salud 2007. Bogotá; 2009.

3. Chow CK, Teo KK, Rangarajan S, Islam S, Gupta R, Avezum A, et al. Prevalence, awareness, treatment, and control of hypertension in rural and urban communities in high-, middle-, and low-income countries. JAMA. 2013;310:959-68.
4. Lira M. Impacto de la hipertensión arterial como factor de riesgo cardiovascular. Revista Médica Clínica Las Condes. 2015;26:156-63.

5. Alhawassi T, Krass I, Pont L. Hypertension in older persons: a systematic review of national and international treatment guidelines. J Clin Hypertens. 2015;7:486-92

6. Xiao J, Hua T, Shen H, Zhang M, Wang XJ, Gao YX, et al. Associations of metabolic disorder factors with the risk of uncontrolled hypertension: a follow-up cohort in rural China. Sci Rep. 2017;7:743-50.

7. Teramoto T, Kawamori R, Miyazaki S, Teramukai S, Sato Y, Okuda Y, et al. Lipid and blood pressure control for the prevention of cardiovascular disease in hypertensive patients: a subanalysis of the OMEGA study. J Atheroscler Thromb. 2014;22:62-75.

8. Fryar C, Ostchega Y, Hales CM, Zhang G, Kruszon-Moran D. Hypertension prevalence and control among adults: United States, 2015-2016. NCHS Data Brief. 2017;(289):1-8

9. Melgarejo J, Maestre GE, Thijs L, Asayama K, Boggia J, Casiglia E, et al. Prevalence, treatment, and control rates of conventional and ambulatory hypertension across 10 populations in 3 continents. Hypertension. 2017;70:50-8.

10. Redon J, Mourad JJ, Schmieder RE, Volpe M, Weiss TW. Why in 2016 are patients with hypertension not $100 \%$ controlled? A call to action. J Hypertens. 2016;34:1480-8.

11. Jhaj R, Gour PR, Kumari S, Sharma S. Association between medication adherence and blood pressure control in urban hypertensive patients in central India. International Journal of Noncommunicable Diseases. 2018;3:9-14.

12. Iglesias A, Alonso JL, Sanz RA, Alonso TV. Factores asociados al control de la presión arterial en la cohorte del estudio del Riesgo de Enfermedad Cardiovascular en Castilla y León (RECCyL). Hipertensión y Riesgo Vascular. 2015;32:48-55.

13. Gee M, Bienek A, McAlister FA, Robitaille C, Joffres M, Tremblay MS, et al. Factors associated with lack of awareness and uncontrolled high blood pressure among Canadian adults with hypertension. Can J Cardiol. 2012;28:375-82.

14. Piercefield EW, Howard ME, Robinson MH, Kirk CE, Ragan AP, Reese SD. Antihypertensive medication adherence and blood pressure control among central Alabama veterans. J Clin Hypertens. 2017;19:543-9.

15. Zhang $Y$, Moran A. Trends in the prevalence, awareness, treatment, and control of hypertension among young adults in the United States, 1999 to 2014. Hypertension. 2017;70:736-42.

16. Jahangiry L, Ghanbari J, Farhangi MA, Sarbakhsh P, Ponnet K. Predictors of poor blood pressure control among Iranian hypertensive patients. BMC Res Notes. 2017;10:668-73.

17. Farah R, Zeidan RK, Chahine MN, Asmar R, Chahine R, Salameh P, et al. Predictors of uncontrolled blood pressure in treated hypertensive individuals: first population凸based study in Lebanon. J Clin Hypertens. 2016;18:871-7.

18. Torres G, Liy J, Delgado M. Programas de hipertensión arterial: gran asistencia y baja adherencia. Rev Colomb Cardiol. 2016;23:578-9.

19. Behnood-rod A, Rabbanifar O, Pourzargar P, Rai A, Saadat Z, Saadat $\mathrm{H}$, et al. Adherence to antihypertensive medications in Iranian patients. Int J Hypertens. 2016;(2016):1-7.

20. Berisa $H$, Dedefo $M$. Non-adherence related factors to antihypertensive medications among hypertensive patients on follow up at Nedjo General Hospital in West Ethiopia. The Open Public Health Journal. 2018;11:62-71.

21. Tocci G, Ferrucci A, Pontremoli R, Ferri C, Rosei EA, Morganti A, et al. Blood pressure levels and control in Italy: comprehensive analysis of clinical data from 2000-2005 and 2005-2011 hypertension surveys. J Hum Hypertens. 2015;29:696-701.

22. Goverwa T, Masuka N, Tshimanga M, Gombe NT, Takundwa L, Bangure D, et al. Uncontrolled hypertension among hypertensive patients on treatment in Lupane District, Zimbabwe, 2012. BMC Res Notes. 2014;7:703-10.

23. Barreto M, Matsuda L, Marcon S. Factors associated with inadequate blood pressure control in patients of primary care. Escola Anna Nery. 2016;20:114-20.

24. Teshome D, Demssie A, Zeleke B. Determinants of blood pressure control amongst hypertensive patients in Northwest Ethiopia. PloS One. 2018;13:1-11.

25. Sarfo F, Mobula LM, Burnham G, Ansong D, Plange-Rhule J, Sarfo-Kantanka $O$, et al. Factors associated with uncontrolled blood pressure among Ghanaians: evidence from a multicenter hospital-based study. PloS One. 2018;13:e0193494.

26. Venkatachalam J, Abrahm SB, Singh Z, Stalin P, Sathya GR. Determinants of patient's adherence to hypertension medications in a rural population of Kancheepuram District in Tamil Nadu, South India. Indian J Community Med. 2015;40:33-8.

27. Silva J, Mantovani M, Kalinke L, Ulbrich E. Hypertension and Diabetes Mellitus Program evaluation on user's view. Rev Bras Enferm. 2015;68:541-7. 